\section{GUN-FIRE DEAFNESS}

BY

\section{E. R. GARNETT PASSE, F.R.C.S. \\ Surgeon Commander, R.N.V.R.}

Since the outbreak of war many naval personnel have complained of deafness immediately following gun fire and bomb explosions. A careful study of these cases has revealed some interesting data.

\section{Incidence}

It occurs at any age and in men without history of previous deafness, familial deafness, or middle-ear infection; also in men without apparent nasal or post-nasal conditions responsible for obstruction of the Eustachian orifices. Most cases are unilateral ; in bilateral cases one ear is affected more than the other.

The type of gun responsible for the majority of cases is the smaller type of 3-inch, 4-inch, 4.7-inch, or salute guns. Deafness does not occur in ratings operating the guns in enclosed turrets, but in those ratings whose duties take them outside the protective shield. The 4.7 -inch guns are the worst offenders.

The number of rounds fired does not seem to matter. In some cases the patients state that they felt pain in the ear or ears at such-and-such a point of the gun fire-this may be after the firing of one shell or several rounds.

The position of the patient at the instant of firing bears some relation to the ear affected. The ear nearest to the mouth of the gun barrel would appear to be the one most frequently affected.

More than half the casualties were wearing cotton-wool plugs, though these may have been hastily and loosely applied. In many cases action has to be taken so rapidly that, although cotton-wool was at hand, no time has been available for the insertion. No case has yet presented itself in which rubber ear plugs have been worn.

\section{Signs and Symptoms}

After the action it appears that the majority of the gun crews are in a brief state of " auditory daze" lasting a varying time from seconds to minutes. During this stage a highpitched ringing sound is invariably noted. In an endeavour to alleviate his deafness the patient may put his finger to his ear, and on withdrawal find that it is blood-stained. He either then or later reports to the sick bay, where, alas! not infrequently the ear is syringed in an endeavour to remove any cerumen or blood present in order to obtain a view of the drum. Giddiness is not complained of. It is often many days before the ship returns to port, when the patient is sent to consult an ear-nose-and-throat specialist. By this time he may present one of four conditions.

1. A more or less rapidly recovering nerve deafness with perfectly normal membrana tympani.

2. A nerve deafness which is recovering but slowly, and in which there is a slightly raised darkish red area on the surface of the membrana extending backwards from the umbo. It is noteworthy that $I$ have not seen this in the anterior or superior portions of the drum.

3. A nerve deafness with a ragged linear tear of the membrana extending in a posterior direction from the umbo. This is often associated with some haemorrhage, seen in the form of dried blood clot on the floor of the auditory canal.

4. A nerve deafness associated with a perforation as in No. 3, plus middle-ear infection. In every case this followed on the ear being syringed.

\section{Course and Duration of Symptoms}

The loss of hearing over the whole range is very marked for the first twenty-four to forty-eight hours, and gradually the hearing returns together with a subsidence of the tinnitus. The upper register is the last to return. A small percentage retrieve their hearing to the same state as before the explosion, but by far the majority recover to varying lesser degrees. Several cases have presented themselves in which the gun-fire deafness was contracted during the last war and has persisted since. As might be predicted, in uncomplicated cases Eustachian inflation gives no material benefit.

From these observations we may deduce that, while no one is immune, gun-fire deafness is more prevalent in the absence of adequate ear plugs and where there is lack of protection from the direct blast of the "back-flash" by a gun shield or, in the case of bomb explosion, by the wall of a house, etc.

Cotton-wool plugs hastily rammed into the ears appear to give quite inadequate protection. It is strongly recommended that rubber ear plugs should be worn, on account of their efficiency, inexpensiveness, and rapidity and ease of application.

It cannot be too strongly stated that if blood is seen in the meatus syringing of the ear or ears is to be avoided until the rent in the membrana has had a chance to heal; this may take as long as fourteen days. In the meantime 2 to 3 drops of guttae sp. vini rect. instilled twice a day are all that is required. If cerumen is present in these cases a little olive oil may be instilled, but no attempt made to remove it by syringing.

Lastly, though complete recovery of hearing is unlikely, reassurance may be given that the greater part of the hearing will return and that the tinnitus will subside.

\section{THE HOSPITAL WORLD STATISTICS FOR 1938}

The latest issue of The Hospitals Year-Book' gives evidence of no wartime thinning. Its twenty-three statistical tables spread themselves over 140 large pages, each table including anything up to seventeen columns, and the diligent student can learn, respecting any one of a thousand voluntary hospitals in the kingdom, the number of available and of daily occupied beds, the number of new in-patients and out-patients, the income and expenditure, and the amount per patient expended on provisions, on surgery and dispensary, on salaries and wages, and on domestic requirements. He may also learn the source of income, in total and per available bed, from voluntary gifts, patients' contributions, public services, investments, and legacies. The compilation suffers from two disadvantages. One is that about 200 hospitals fail to conform to certain minimum conditions in the way they present their accounts, and these have to be excluded from the statistical tables; the other is that the statistics relate to 1938 , which seems a great deal more than two years ago. Although a pre-war year it was an unfortunate one for voluntary hospitals. The total expenditure was roughly $£ 20,200,000$, and the income $£ 20,000,000$ "result misery," as Mr. Micawber would say. This is the first deficit in a ten-year period. In 1934 there was a surplus of one and a half millions, in 1935 a surplus of one million, and in 1936 and 1937 surpluses of about half a million. It is true that the deficit for 1938 is only small; but it is an ominous advent for 1939, when there was a vast dislocation of normal hospital progress. It is mainly the large London hospitals, with 100 or more beds, which show deficits. Forty such hospitals have a deficit between them of $£ 240,000$; sixteen others show surpluses, but the total does not reach $\$ 100,000$. The provincial hospitals are better off. They still in total have a surplus, though it is less than it was the year before.

\section{Regionalization of Hospitals}

One section of the Year-Book is devoted to the very live subject of regionalization, and descriptions are given 'The Hospitals Year-Book, 1940 . Edited by $\mathrm{R} . \mathrm{H}$. P. Orde, O.B.L. 